\title{
RECURRENT GEODESICS IN FLAT LORENTZ 3-MANIFOLDS
}

\author{
VIRGINIE CHARETTE, WILLIAM M. GOLDMAN AND CATHERINE A. \\ JONES
}

\begin{abstract}
We introduce the notion of recurrent geodesic rays in a complete flat Lorentz 3-manifold. We completely classify the dynamical behavior of geodesics in cyclic quotients, and apply this classification to more general quotients. In particular we show that for any pair of closed geodesics $\gamma_{1}, \gamma_{2}$ there exists a unique geodesic which spirals around $\gamma_{1}$ in forward time and $\gamma_{2}$ in backwards time.
\end{abstract}

\section{Contents}

1. Introduction 1

2. Geometry of Minkowski spacetime 2

2.1. Minkowski $2+1$-Spacetime 2

2.2. Isometries 3

2.3. Stable and unstable planes 4

2.4. Geodesics 4

3. Cylinders 5

4. Recurrent geodesics in flat Lorentz 3-manifolds $\quad 10$

References 11

\section{INTRODUCTION}

In this paper, we investigate dynamical properties of geodesics in flat Lorentz 3-manifolds $M$. We assume $M$ is geodesically complete, that is, $M$ is the quotient $\mathbb{A}^{2,1} / \Gamma$ of 3 -dimensional Minkowski spacetime $\mathbb{A}^{2,1}$ by a discrete group $\Gamma$ of affine isometries acting properly on $\mathbb{A}^{2,1}$.

The first section develops preliminaries on Minkowski space and its isometries. Section 3 is devoted to the particular case of cylinders: quotients of spacetime by a cyclic hyperbolic group $\langle\gamma\rangle$. Such basic examples already display rich and interesting behavior. By means of a

Date: August 2, 2001.

The authors gratefully acknowledge partial support from National Science Foundation grant DMS-9803518. 
$\langle\gamma\rangle$-invariant function, we show that recurrent geodesic rays (which are the images of nonproper maps) must lie in one of two codimension-one submanifolds. Further, since not every geodesic ray in those submanifolds is recurrent, we give a characterization (in terms of their directing vectors) of recurrent geodesic rays in cylinders. The notion of a geodesic ray spiralling around a closed geodesic is introduced.

The most interesting examples are Margulis spacetimes, when $\Gamma$ is a free purely hyperbolic discrete subgroup of the isometry group of $\mathbb{A}^{2,1}$, that is a Schottky group. Drumm [2,3] showed that every noncocompact discrete subgroup of $\mathrm{SO}(2,1)$ admits proper affine deformations. Nonclosed birecurrent geodesics can only be found when the rank of the fundamental group is greater than one.

The main results of this paper may also be found in [1].

\section{Geometry of Minkowski spacetime}

2.1. Minkowski $2+1$-Spacetime. Let $\mathbb{R}^{2,1}$ denote a three-dimensional real vector space equipped with the standard symmetric bilinear form of signature $(2,1)$ :

$$
\mathbb{B}(\mathrm{x}, \mathrm{y})=x_{1} y_{1}+x_{2} y_{2}-x_{3} y_{3},
$$

where $\mathbf{x}=\left(x_{1}, x_{2}, x_{3}\right)$ and $\mathbf{y}=\left(y_{1}, y_{2}, y_{3}\right)$. A vector $\mathbf{v} \in \mathbb{R}^{2,1}$ is spacelike (resp. timelike, lightlike) if $\mathbb{B}(\mathrm{v}, \mathrm{v})>0$ (resp. $\mathbb{B}(\mathrm{v}, \mathrm{v})<0, \mathbb{B}(\mathrm{v}, \mathrm{v})=0)$. (Lightlike vectors are also called null.)

Denote by $\mathbb{A}^{2,1}$ the affine space modeled on $\mathbb{R}^{2,1}$ : for every $p \in \mathbb{A}^{2,1}$, the tangent space

$$
\mathbb{A}_{p}^{2,1}=\left\{q-p: q \in \mathbb{A}^{2,1}\right\}
$$

is endowed with the bilinear form $\mathbb{B}(\cdot, \cdot)$. Clearly, $\mathbb{A}_{p}^{2,1} \cong \mathbb{R}^{2,1}$.

Any line in $\mathbb{A}^{2,1}$ can be described as $p+\mathbb{R} v$, where $p \in \mathbb{A}^{2,1}$ and $\mathbf{v} \in \mathbb{R}^{2,1}$. Two lines $p+\mathbb{R} v, q+\mathbb{R} \mathrm{w}$ are parallel if $\mathbf{v}=k \mathrm{w}$ for some $k \neq 0$. The line $p+\mathbb{R} v$ is called spacelike, timelike or lightlike according to the causal character of $\mathrm{v}$.

The set of non-spacelike vectors, with the origin removed, has two connected components. A choice of component is a time orientation on $\mathbb{R}^{2,1}$. We will adopt the standard time orientation: a non-spacelike vector $\mathrm{v}=\left(v_{1}, v_{2}, v_{3}\right)$ is future-pointing if $v_{3}>0$ and past-pointing otherwise.

The Lorentz-orthogonal plane of $\mathrm{v} \in \mathbb{R}^{2,1}$ at $p$ is the set of all vectors based at $p$ which are Lorentz-orthogonal to v:

$$
\begin{aligned}
p+\mathrm{v}^{\perp} & =\left\{q \in \mathbb{A}^{2,1}: \mathbb{B}(q-p, \mathrm{v})=0\right\} \\
& =\left\{p+\mathbf{x}: \mathbb{B}(\mathbf{x}, \mathbf{v})=0, \mathbf{x} \in \mathbb{R}^{2,1}\right\} .
\end{aligned}
$$


2.2. Isometries. An affine isometry of $\mathbb{A}^{2,1}$ is an affine transformation $\gamma$ whose linear part preserves the bilinear form $\mathbb{B}(\cdot, \cdot)$. Thus the linear part of an affine isometry of $\mathbb{A}^{2,1}$ lies in $\mathrm{O}(2,1)$. The isometry group of $\mathbb{A}^{2,1}$ is denoted $\operatorname{Isom}\left(\mathbb{A}^{2,1}\right)$. The connected component of the identity of $\mathrm{O}(2,1)$, denoted $\mathrm{SO}(2,1)^{0}$, consists of those linear isometries which preserve orientation and time orientation.

An affine isometry is said to be hyperbolic if its linear part is hyperbolic: that is, it is an element of $\mathrm{SO}(2,1)^{0}$ which has three real distinct eigenvalues.

If $g \in \mathrm{SO}(2,1)^{0}$ is hyperbolic, then its eigenvalues are $\lambda<1<\lambda^{-1}$, for some positive $\lambda \in \mathbb{R}$. The $\lambda$ - and $\lambda^{-1}$-eigendirections are lightlike and the 1-eigendirection is spacelike.

Let $g \in \mathrm{SO}(2,1)^{0}$ be a hyperbolic isometry with smallest eigenvalue $\lambda<1$. Set $\mathbf{x}^{+}(g), \mathbf{x}^{-}(g)$ to be future-pointing eigenvectors of $\lambda^{-1}$, $\lambda$, respectively, normalized so that $\left\|\mathrm{x}^{ \pm}(g)\right\|=1$, where $\|\cdot\|$ denotes Euclidean length.

Choose $\mathbf{x}^{0}(g)$ to be the unique spacelike 1-eigenvector, satisfying $\mathbb{B}\left(\mathbf{x}^{0}(g), \mathbf{x}^{0}(g)\right)=1$, such that the null frame

$$
\left\{\mathrm{x}^{0}(g), \mathbf{x}^{-}(g), \mathbf{x}^{+}(g)\right\}
$$

is a positively oriented basis. For hyperbolic $\gamma \in \operatorname{Isom}\left(\mathbb{A}^{2,1}\right)$ with linear part $g$, set:

$$
\left\{\mathrm{x}^{0}(\gamma), \mathrm{x}^{-}(\gamma), \mathrm{x}^{+}(\gamma)\right\}=\left\{\mathrm{x}^{0}(g), \mathrm{x}^{-}(g), \mathrm{x}^{+}(g)\right\}
$$

The following facts are well known:

Lemma 2.1. Let $\gamma \in \operatorname{Isom}\left(\mathbb{A}^{2,1}\right)$ be hyperbolic. Then there exists a line $C_{\gamma} \subset \mathbb{A}^{2,1}$, parallel to $\mathrm{x}^{0}(\gamma)$, which is invariant under the action of $\gamma$. Moreover, $\gamma$ acts by translation on $C_{\gamma}$. Furthermore, $C_{\gamma}$ is the unique $\gamma$-invariant line if and only if $\gamma$ acts freely on $\mathbb{A}^{2,1}$.

Proof. Since $\gamma$ is affine, it acts on $N$, the space of lines parallel to $\mathbf{x}^{0}(\gamma)$. Observe that $N$ is isomorphic to the Lorentz-orthogonal plane $x^{0}(\gamma)^{\perp}$, which, in turn, is isomorphic to two-dimensional Minkowski space. The eigenvalues of the induced action of $\gamma$ are each different from 1 , therefore this action has a fixed point.

Let $C_{\gamma} \subset \mathbb{A}^{2,1}$ be the line parallel to $x^{0}(\gamma)$ corresponding to this fixed point in $N$; clearly, $C_{\gamma}$ is invariant under the action of $\gamma$ : if $p \in C_{\gamma}$,

$$
\gamma(p)=p+\alpha \mathrm{x}^{0}(\gamma)
$$

where $\alpha \in \mathbb{R}$. Any other point $q$ on $C_{\gamma}$ can be written as $p+t \mathrm{x}^{0}(\gamma)$, for some $t \in \mathbb{R}$. Since $\mathbf{x}^{0}(\gamma)$ is fixed by $\gamma$ :

$$
\gamma(q)=\gamma\left(p+t \mathrm{x}^{0}(\gamma)\right)=\gamma(p)+t \mathrm{x}^{0}(\gamma)=q+\alpha \mathrm{x}^{0}(\gamma)
$$


Thus $\gamma$ acts by translation on $C_{\gamma}$.

Finally, $\gamma$ fixes a point $p$ if and only if the line $p+\mathbb{R} x^{0}(\gamma)$ is pointwise fixed, so $C_{\gamma}$ is the unique $\gamma$-invariant line if and only if $\gamma$ acts freely.

Definition 2.2. For a hyperbolic isometry $\gamma \in \operatorname{Isom}\left(\mathbb{A}^{2,1}\right)$, set $\alpha(\gamma)$ to be the parameter $\alpha$ in Equation (1). Then $\alpha$, a signed measure of Lorentzian length, is called the Margulis invariant.

This function was introduced by Margulis [4, 5] to show that an affine group whose linear part is a Schottky group could act properly discontinuously on $\mathbb{A}^{2,1}$. Since $\alpha(\gamma)$ is the translation factor along $C_{\gamma}$, the proof of Lemma 2.1 implies:

Lemma 2.3. Let $\gamma \in \operatorname{Isom}\left(\mathbb{A}^{2,1}\right)$ be a hyperbolic isometry. Then $\gamma$ acts freely on $\mathbb{A}^{2,1}$ if and only if $\alpha(\gamma) \neq 0$.

\subsection{Stable and unstable planes.}

Definition 2.4. Let $\gamma \in \operatorname{Isom}\left(\mathbb{A}^{2,1}\right)$ be hyperbolic and let $p \in C_{\gamma}$. The planes

$$
\begin{aligned}
& E_{\gamma}^{+}=p+\left\langle\mathrm{x}^{0}(\gamma), \mathrm{x}^{+}(\gamma)\right\rangle \\
& E_{\gamma}^{-}=p+\left\langle\mathrm{x}^{0}(\gamma), \mathrm{x}^{-}(\gamma)\right\rangle,
\end{aligned}
$$

are respectively called the weak-unstable plane and the weak-stable plane of $\gamma$.

Note that $E_{\gamma}^{+}=E_{\gamma^{-1}}^{-}, E_{\gamma}^{+} \cap E_{\gamma}^{-}=C_{\gamma}$, and $E_{\gamma}^{ \pm}$is $\gamma$-invariant.

Consider the orbit of a point $q$ in $\mathbb{A}^{2,1}$, under the action of $\gamma$. We can write

$$
q=p+A^{+} \mathbf{x}^{+}(g)+A^{-} \mathbf{x}^{-}(g),
$$

where $p \in C_{\gamma}$ and $A^{ \pm} \in \mathbb{R}$. Thus for every $n$,

$$
\gamma^{n}(q)=p+n \alpha(\gamma) \mathbf{x}^{0}(g)+A^{+} \lambda^{-n} \mathbf{x}^{+}(g)+A^{-} \lambda^{n} \mathbf{x}^{-}(g) .
$$

If $A^{+}=0$, the orbit converges towards $C_{\gamma}$. When $A^{+} \neq 0$, the sequence approaches $E^{+}$, but eventually leaves every compact set intersecting the weak-unstable plane.

2.4. Geodesics. Let $M=\mathbb{A}^{2,1} / \Gamma$, where $\Gamma<\operatorname{Isom}\left(\mathbb{A}^{2,1}\right)$ acts properly on $\mathbb{A}^{2,1}$. Then $M$ is a complete Lorentz manifold and its fundamental group is isomorphic to $\Gamma$. Let $\pi: \mathbb{A}^{2,1} \longrightarrow M$ denote the quotient projection. 
A geodesic in $M$ is a nonconstant affine map $l: \mathbb{R}^{+} \longrightarrow M$, that is, the composition $\pi \circ \tilde{l}$ where $\tilde{l}: \mathbb{R} \longrightarrow \mathbb{A}^{2,1}$ is a nonconstant affine map. The reverse of a geodesic $l$ is the geodesic $-l$ defined by

$$
-l(t):=l(-t)
$$

A geodesic ray in $M$ is a nonconstant affine map $l: \mathbb{R}^{+} \longrightarrow M$, that is, the composition $\pi \circ \tilde{l}$ where $\tilde{l}: \mathbb{R}^{+} \longrightarrow \mathbb{A}^{2,1}$ is a nonconstant affine map. The forward ray of a geodesic $l$ is the restriction of $l$ to $\mathbb{R}^{+}$and the backward ray of $l$ is the forward ray of $-l$.

A geodesic ray $l$ is parallel to a line, vector or ray, if its direction vector $\mathrm{v}$ (defined by $l(t)=q+t \mathrm{v}$ ) is parallel to the line, vector or ray.

A geodesic $l$ is periodic if for some $T>0$, and all $t>0$,

$$
l(t+T)=l(t) .
$$

We will often identify a periodic geodesic with its image, which is an immersed $S^{1}$ in $M$. The cylinder associated to $l$ is the covering space $M_{l} \longrightarrow M$ induced by the equivalence class of $l$ in $\pi_{1}(M, l(0))$.

The following conditions are equivalent:

- $l$ is a proper map: for every compact $K \subset M$, the inverse image $l^{-1}(K) \subset \mathbb{R}^{+}$is compact;

- For every increasing sequence $t_{k} \longrightarrow+\infty$, the sequence $l\left(t_{k}\right)$ has no accumulation point;

- $l$ is not periodic and the image $l\left(\mathbb{R}^{+} \cup\{0\}\right)$ is closed.

A geodesic ray $l$ is recurrent if the mapping $l: \mathbb{R}^{+} \longrightarrow M$ is not proper. Equivalently, its image $l\left(\mathbb{R}^{+}\right)$has compact closure in $M$.

For example, a closed geodesic is recurrent.

A geodesic ray $r$ spirals around a periodic geodesic $l$ if for every neighborhood $N$ of $l$, there exists $T=T(N)>0$ such that $r(t) \in N$ for $t>T$. In particular, such a geodesic is recurrent.

A geodesic $l$ is birecurrent if both its forward ray and its backward ray recur. A geodesic $l$ bispirals if both its forward ray and backward ray spiral around closed geodesics.

\section{Cylinders}

We now classify geodesics in quotients $\mathbb{A}^{2,1} /\langle\gamma\rangle$, where $\gamma$ is a hyperbolic affine isometry of $\mathbb{A}^{2,1}$. the cyclic group it generates. In this section $M$ will denote the quotient $\mathbb{A}^{2,1} /\langle\gamma\rangle$.

The stable surface $M^{-}=\pi\left(E_{\gamma}^{-}\right)$and the unstable surface $M^{+}=$ $\pi\left(E_{\gamma}^{+}\right)$are each diffeomorphic to an annulus to which $M$ deformation retracts. Similarly $M^{+}, M^{-}$each deformation retract to the core geodesic $M^{0}=M^{+} \cap M^{-}$, which is the unique closed geodesic in $M$. 
Lemma 3.1. A recurrent geodesic ray $\mathbb{R}^{+} \longrightarrow M$ lies in either $M^{+}$or $M^{-}$.

Proof. Let $p$ be an arbitrary point on the invariant line $C_{\gamma}$. For every point $q \in \mathbb{A}^{2,1}$, write

$$
q=p+A^{0} \mathbf{x}^{0}(\gamma)+A^{-} \mathbf{x}^{-}(\gamma)+A^{+} \mathbf{x}^{+}(\gamma)
$$

where $A^{-}, A^{+}, A^{0} \in \mathbb{R}$ and $\left.\left\{\mathrm{x}^{-}(\gamma)\right), \mathrm{x}^{+}(\gamma), \mathrm{x}^{0}(\gamma)\right\}$ is the null frame associated to $\gamma$. Then

$$
\gamma(q)=p+\left(A^{0}+\alpha(\gamma)\right) \mathbf{x}^{0}(\gamma)+\lambda A^{-} \mathbf{x}^{-}(\gamma)+\lambda^{-1} A^{+} \mathbf{x}^{+}(\gamma)+.
$$

and

$$
\mathbb{B}\left(\gamma(q)-p, \mathrm{x}^{ \pm}(\gamma)\right)=\mathbb{B}\left(\gamma(q-p), \mathrm{x}^{ \pm}(\gamma)\right)=\lambda^{ \pm 1} \mathbb{B}\left(q-p, \mathrm{x}^{ \pm}(\gamma)\right) .
$$

Thus the quadratic function $\tilde{f}: \mathbb{A}^{2,1} \longrightarrow \mathbb{R}$ given by

$$
\tilde{f}(q)=\mathbb{B}\left(q-p, \mathrm{x}^{-}(\gamma)\right) \mathbb{B}\left(q-p, \mathrm{x}^{+}(\gamma)\right)
$$

is independent of the choice of $q \in C_{\gamma}$, and is $\langle\gamma\rangle$-invariant. Define $f: M \longrightarrow \mathbb{R}$ as $f:=\pi \circ \tilde{f}$.

Suppose that $l: \mathbb{R}^{+} \longrightarrow M$ is a recurrent geodesic ray. Write $l=\pi \circ \tilde{l}$ where

$$
\begin{aligned}
\tilde{l}: \mathbb{R}^{+} & \longrightarrow \mathbb{A}^{2,1} \\
t & \longmapsto q+t \mathrm{v}
\end{aligned}
$$

is the corresponding geodesic ray in $\mathbb{A}^{2,1}$. Then

$$
(f \circ l)(t)=(\tilde{f} \circ \tilde{l})(t)=a+b t+c t^{2}
$$

where $p$ is an arbitrary point on $C_{\gamma}$ and

$$
\begin{aligned}
a & =f(l(0)) \\
b & =\mathbb{B}\left(q-p, \mathrm{x}^{+}(\gamma)\right) \mathbb{B}\left(\mathrm{v}, \mathrm{x}^{-}(\gamma)\right)+\mathbb{B}\left(q-p, \mathrm{x}^{-}(\gamma)\right) \mathbb{B}\left(\mathrm{v}, \mathrm{x}^{+}(\gamma)\right) \\
c & =\mathbb{B}\left(\mathrm{v}, \mathrm{x}^{+}(\gamma)\right) \mathbb{B}\left(\mathrm{v}, \mathrm{x}^{-}(\gamma)\right) .
\end{aligned}
$$

Unless $c=0$, the function $f \circ l: \mathbb{R}^{+} \rightarrow \mathbb{R}$ in (4) is quadratic and tends to $\pm \infty$ as $t \longrightarrow+\infty$. If $c=0$ but $b \neq 0$, then $f \circ l$ is a nonconstant affine function, also tending to $\pm \infty$ as $t \longrightarrow+\infty$. In either case $f \circ l$ defines a proper map $\mathbb{R}^{+} \longrightarrow \mathbb{R}$, contradicting recurrence of $l$. Thus $f \circ l$ is constant, that is $b=c=0$.

Since $c=0$, at least one of $\mathbb{B}\left(\mathrm{v}, \mathrm{x}^{+}(\gamma)\right), \mathbb{B}\left(\mathrm{v}, \mathrm{x}^{-}(\gamma)\right)$ must vanish. Thus $\tilde{l}$ is parallel to either $E^{-}$or $E^{+}$respectively. If it is parallel to both, then $v$ is parallel to $x^{0}(\gamma)$. We postpone the discussion of this case to the end of the proof. 
Suppose that $\tilde{l}$ is parallel to $E^{+}$, but not $E^{-}$. Thus $\mathbb{B}\left(\mathrm{v}, \mathrm{x}^{+}(\gamma)\right)=0$ but $\mathbb{B}\left(\mathbf{v}, \mathbf{x}^{-}(\gamma)\right) \neq 0$. Then $b=0$ implies that $\mathbb{B}\left(q-p, \mathbf{x}^{+}(\gamma)\right)=0$. Therefore $\tilde{l}: \mathbb{R}^{+} \longrightarrow E^{+}$.

In the same fashion, if $\tilde{l}$ is parallel to $E^{-}$, but not $E^{+}$, then $\tilde{l}\left(\mathbb{R}^{+}\right) \subset$ $E^{-}$.

Finally, suppose that $\tilde{l}$ is parallel to $x^{0}(\gamma)$, but lies on neither $E^{-}$nor $E^{+}$. Let

$$
U=\mathbb{A}^{2,1}-\left(E^{-} \cup E^{+}\right) .
$$

Consider the quotient space $N$ of $\mathbb{A}^{2,1}$ by the one-dimensional foliation parallel to $C_{\gamma}$. The restriction of the quotient map $\Pi: \mathbb{A}^{2,1} \longrightarrow N$ to $U$ is a $\langle\gamma\rangle$-equivariant mapping with respect to a proper action of $\langle\gamma\rangle \cong \mathbb{Z}$ on $U^{\prime}=\Pi(U)$. Specifically, $U^{\prime}$ is the complement of two intersecting lines in the plane $N$ and $\langle\gamma\rangle$ acts by hyperbolic linear maps with discrete orbits.

Thus the composition

$$
\begin{aligned}
\langle\gamma\rangle \times\{q\} & \longrightarrow \mathbb{A}^{2,1} \stackrel{\Pi}{\longrightarrow} N \\
\left(\gamma^{n}, q\right) & \longmapsto \gamma^{n} q
\end{aligned}
$$

is proper. Consequently the mapping

$$
\begin{aligned}
\langle\gamma\rangle \times \mathbb{R}^{+} & \longrightarrow \mathbb{A}^{2,1} \\
\left(\gamma^{n}, t\right) & \longmapsto \gamma^{n}(q+t \mathrm{v})
\end{aligned}
$$

is proper. Therefore $l: \mathbb{R}^{+} \longrightarrow M$ is proper, a contradiction.

Lemma 3.2. Suppose $l$ is a geodesic ray as defined in (3). Suppose one of the following conditions hold:

(i) $l \subset M^{+}$and

$$
\mathbb{B}\left(\alpha(\gamma) \mathbf{x}^{0}(\gamma), \mathbf{v}\right)>0
$$

(ii) $l \subset M^{-}$and

$$
\mathbb{B}\left(\alpha(\gamma) \mathbf{x}^{0}(\gamma), \mathrm{v}\right)<0
$$

Then for every $p \in C_{\gamma}, \pi(p) \subset M^{0}$ is an accumulation point of $l$.

Proof. Write $l=\pi \circ \tilde{l}$ as above. We first show (i); the proof of (ii) is completely analogous. Suppose then that $l \subset M^{+}$, so that $\tilde{l}$ lies in the weak-unstable plane $E^{+}$. Let $\widetilde{V}$ be a neighborhood of $p$ such that $\widetilde{V} \cap E^{+}$is bounded by $p \pm k^{0} \mathrm{x}^{0}(\gamma) \pm k^{+} \mathrm{x}^{+}(\gamma)$. Then:

$$
\gamma^{n} \widetilde{V} \cap E^{+}=p+\left(\alpha(\gamma) \pm k^{0}\right) \mathbf{x}^{0}(\gamma) \pm k^{+} \lambda^{-n} \mathbf{x}^{+}(\gamma)
$$

Thus the $\gamma^{n}$-translates of $\widetilde{V}$ are dilated in the $\mathrm{x}^{+}(\gamma)$-direction at the rate of $\lambda^{-n}$, whereas the $\mathbf{x}^{+}(\gamma)$-coefficient of $\tilde{l}$ grows linearly. Thus for 
large enough $n$, every $\gamma^{n}(\widetilde{V})$ intersects $\tilde{l}$ and $p$ is an accumulation point of $l$.

The restriction of $\gamma$ to $E^{+}$is represented by the affine map

$$
\gamma^{+}:\left[\begin{array}{l}
x \\
y
\end{array}\right] \longmapsto\left[\begin{array}{cc}
1 & 0 \\
0 & \lambda^{-1}
\end{array}\right]\left[\begin{array}{l}
x \\
y
\end{array}\right]+\left[\begin{array}{l}
\alpha \\
0
\end{array}\right]
$$

where $0<\lambda<1$ and $\alpha \neq 0$.

Apply the coordinate change

$$
\left[\begin{array}{l}
x \\
y
\end{array}\right] \longrightarrow\left[\begin{array}{l}
x \\
\eta
\end{array}\right]
$$

where

$$
\begin{aligned}
& \eta(x, y)=\lambda^{x / \alpha} y \\
& y(x, \eta)=\lambda^{-x / \alpha} \eta .
\end{aligned}
$$

The action of $\langle\gamma\rangle$ in $(x, \eta)$-coordinates is given by horizontal translation by $n \alpha$ :

$$
\left(\gamma^{+}\right)^{n}:\left[\begin{array}{l}
x \\
\eta
\end{array}\right] \longmapsto\left[\begin{array}{c}
x+n \alpha \\
\eta
\end{array}\right] .
$$

This defines a $\gamma$-invariant diffeomorphism of the stable surface

$$
\begin{aligned}
& \xi: M^{+} \longrightarrow \mathbb{R} / \alpha \mathbb{Z} \times \mathbb{R} \\
& {\left[\begin{array}{l}
x \\
y
\end{array}\right] \longmapsto\left[\begin{array}{cc}
x & \bmod \alpha \mathbb{Z} \\
\eta(x, y)
\end{array}\right] . }
\end{aligned}
$$

A geodesic in the unstable plane falls into one of two categories, depending on whether it is parallel to the eigenvector $x^{+}(\gamma)$ or not. If the geodesic is parallel to $\mathbf{x}^{+}(\gamma)$, then we call it a vertical geodesic.

Theorem 3.3. A geodesic ray l (as defined by (3)) in $M$ is recurrent if and only if one of the following holds:

(i) $l \subset M^{+}$and $\mathbb{B}\left(\alpha(\gamma) \mathrm{x}^{0}(\gamma)\right.$, v) $>0$

(ii) $l \subset M^{-}$and $\mathbb{B}\left(\alpha(\gamma) x^{0}(\gamma), v\right)<0$.

Proof. We shall show (i). Since in $(x, \eta)$-coordinates, $\gamma$ acts by translation along the $x$-axis, a geodesic ray is proper if and only if its $\eta$ coordinate is unbounded. Let $\beta: \mathbb{R}^{+} \longrightarrow M^{+}$be a geodesic in the unstable surface and choose a lift $\tilde{\beta}: \mathbb{R}^{+} \longrightarrow E^{-}$.

If $\tilde{\beta}$ is vertical, it admits the following representation:

$$
\tilde{\beta}(t)=\left[\begin{array}{l}
x_{0} \\
y_{0}
\end{array}\right]+t\left[\begin{array}{l}
0 \\
c
\end{array}\right] .
$$


In the $(x, \eta)$-coordinate system:

$$
\xi \circ \tilde{\beta}(t)=\left[\begin{array}{cc}
x_{0} & \bmod \alpha \mathbb{Z} \\
& \eta_{0}+c^{\prime} t
\end{array}\right]
$$

where

$$
\begin{aligned}
\eta_{0} & =\lambda^{x_{0} / \alpha} y_{0} \\
c^{\prime} & =\lambda^{x_{0} / \alpha} c .
\end{aligned}
$$

As $t \longrightarrow+\infty$, the $\eta$-coordinate grows without bound and thus the geodesic ray $\xi \circ \tilde{\beta}(t)$ does not recur.

If $\beta$ is not vertical, then

$$
\tilde{\beta}(t)=\left[\begin{array}{l}
x_{0} \\
y_{0}
\end{array}\right]+t\left[\begin{array}{c}
1 \\
m
\end{array}\right]
$$

where $m \in \mathbb{R}$ is the slope of $\tilde{\beta}$. Then

$$
\eta \circ \tilde{\beta}(t)=e^{\mu t}\left(y_{0}^{\prime}+t m^{\prime}\right)
$$

where

$$
\begin{aligned}
y_{0}^{\prime} & =\lambda^{x_{0} / \alpha} y_{0} \\
m^{\prime} & =\lambda^{x_{0} / \alpha} m \\
\mu & =\log (\lambda) / \alpha
\end{aligned}
$$

The $\eta$-coordinate is bounded exactly when $\mu<0$, that is, when $\alpha<0$ (since $0<\lambda<1$ ).

Otherwise $e^{\mu t}>1$ and $\beta$ does not recur.

(This theorem follows from a similar result in [1], using the fact that the weak-stable plane of a hyperbolic isometry is the weak-unstable plane of its inverse.)

Figure 1 illustrates the proof of the theorem, by showing the orbit of a recurrent geodesic in $(x, \eta)$-coordinates. The geodesic $\beta \subset \mathcal{K}^{+}$may cross the closed geodesic $M^{0}$. In that case $\beta$ crosses $M^{0}$ transversely, and then spirals back towards $M^{0}$, intersecting itself infinitely many times.

If $m=0$, then $\beta$ never intersects itself, but approaches $M^{0}$ asymptotically from one side. (See Figure 2.)

Corollary 3.4. The only birecurrent geodesic in $M$ is the closed geodesic $M^{0}$. 


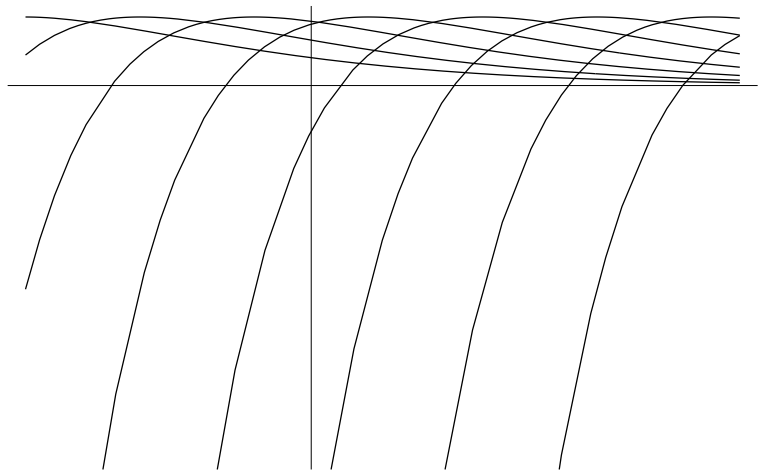

FiguRE 1. The orbit of a recurrent geodesic on the unstable surface in $(x, \eta)$-coordinates: the horizontal line is $M^{0}$, which all recurrent geodesics in $M^{+}$approach asymptotically.

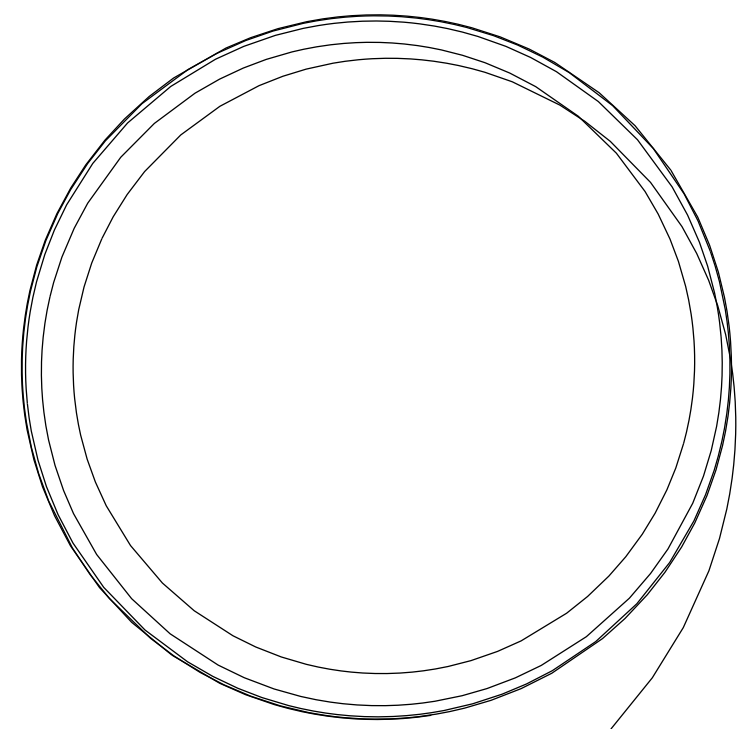

Figure 2. The behavior of a ray spiralling towards a closed geodesic.

\section{RECURRENT GEODESICS IN FLAT LORENTZ 3-MANifOLDS}

Let $M$ be a Margulis spacetime; recall that its fundamental group is purely hyperbolic. Let $l_{1}, l_{2}$ be (oriented) closed geodesics in $M$. Then there exists a birecurrent geodesic $l$ whose forward ray spirals towards $l_{1}$ and whose backward ray spirals towards $l_{2}$. Such geodesics correspond to equivalence classes of arcs $a$ whose endpoints lie on $l_{1}$ 
and $l_{2}$, under the equivalence relation defined by homotopies keeping the endpoints on $l_{1}$ and $l_{2}$ respectively (a homotopy relative to $l_{1}$ and $\left.l_{2}\right)$.

Choose a basepoint $x \in M$ and the corresponding universal covering space $\tilde{M} \longrightarrow M$ and developing map dev $: \tilde{M} \longrightarrow \mathbb{A}^{2,1}$. Join $l_{1}, l_{2}$ to $x$ by $\operatorname{arcs} a_{1}, a_{2}$ respectively such that the composition $a_{1} a_{2}^{-1}$ is homotopic to $a$ by a homotopy relative to $l_{1}$ and $l_{2}$. Let $\gamma_{i}$ be the holonomy of the based loops corresponding to $l_{i}, i=1,2$. Thus $l_{i}$ lifts to $C_{\gamma_{i}}$ in the universal cover.

For $\gamma \in \Gamma$, denote its associated stable and unstable surfaces in $M$ by $M_{\gamma}^{ \pm}$. Every geodesic in $M$ is the projection of a geodesic in the cylinder $M_{C_{\gamma}}$, since it is a covering space. In particular, Theorem 3.3 implies:

Proposition 4.1. Let $l$ be a geodesic which lies in $M_{\gamma_{i}}^{+}$. Then the forward ray of $l$ spirals around $l_{i}$.

Theorem 4.2. If a geodesic $l \subset M$ lies in either $M_{\gamma}^{+}$or $M_{\gamma}^{-}$, where $\gamma \in \Gamma$, then $l$ is recurrent.

We obtain birecurrent geodesics in the following manner. Consider:

$$
l=M_{\gamma_{1}}^{+} \cap M_{\gamma_{2}}^{+} .
$$

Write its inverse image $\tilde{l}$ in $\mathbb{A}^{2,1}$ as $\tilde{l}(t)=p+t \mathrm{v}$. Then:

$$
\mathbb{B}\left(\mathrm{v}, \alpha\left(\gamma_{1}\right) \mathrm{x}^{0}\left(\gamma_{1}\right)\right) \mathbb{B}\left(\mathrm{v}, \alpha\left(\gamma_{2}\right) \mathrm{x}^{0}\left(\gamma_{2}\right)\right)<0 .
$$

Each condition of Theorem 3.3 applies, one in each direction of $\tilde{l}$, implying that $l$ is birecurrent.

We obtain a similar result for the intersection of stable surfaces, since $E_{\gamma}^{-}=E_{\gamma^{-1}}^{+}$.

\section{REFERENCES}

[1] Charette, V., "Proper Actions of Discrete Groups in 2+1 Spacetime," Doctoral dissertation, University of Maryland (2000).

[2] Drumm, T., Fundamental polyhedra for Margulis space-times, Doctoral Dissertation, University of Maryland (1990).

[3] _ Fundamental polyhedra for Margulis space-times, Topology 31 (4) (1992), 677-683.

[4] Margulis, G. A., Free properly discontinuous groups of affine transformations, Dokl. Akad. Nauk SSSR 272 (1983), 937-940

[5] _ Complete affine locally flat manifolds with a free fundamental group, J. Soviet Math. 134 (1987), 129-134 
Department of Mathematics and Statistics, McMaster University, Hamilton, Ontario, Canada, L8S 4K1

Department of Mathematics, University of Maryland, College Park, MD 20742

E-mail address: Charette: charette@math.mcmaster.ca

Goldman: wmg@math.umd.edu, Jones: caj@math.umd.edu 